\title{
Impact of grain-coating iron minerals on dielectric response of quartz sand and implications for ground-penetrating radar
}

\author{
Matthew Josh ${ }^{1}$, Melvyn J. Lintern ${ }^{1}$, Anton W. Kepic ${ }^{2}$, and Mike Verrall
}

\begin{abstract}
An unexpected result of ground-penetrating radar (GPR) surveys in the Great Victoria Desert (South Australia) was the lack of returning signal in what appeared to be a favorable environment for GPR, with dry silica sand and calcrete aggregates in the near surface. We found that the dielectric response of the dry sand samples had much higher dielectric losses than comparable sands from Western Australia and that the dielectric losses are controlled by the presence of iron oxide minerals, although iron concentrations themselves are only around $0.4 \%$. The samples contained over $90 \%$ quartz, with subsidiary amounts of carbonates, kaolin, and smectite occurring with the iron oxide minerals as a coating on the quartz grains. An acid washing procedure removed the reducible iron oxide minerals from the clay coating but left the clays substantially unaltered. Subsequent dielectric and magnetic analysis of the samples indicates that the iron oxide minerals removed during the washing process are responsible for the reduction of GPR penetration at $250 \mathrm{MHz}$ from approximately $10 \mathrm{~m}$ to only $1 \mathrm{~m}$.
\end{abstract}

\section{INTRODUCTION}

Occasions where ground-penetrating radar (GPR) has failed to provide useful data are not often published in the literature, but it is generally understood among GPR practitioners that there are limited environments where the technique performs reliably to provide useful geophysical data (Daniels et al., 1988; Davis and Annan, 1989; Doolittle and Collins, 1995; Weaver, 2006). The limitations for GPR depth of investigation are commonly related to the electromagnetic properties of the formation of interest, in particular, the presence of saline water or clay minerals, which strongly attenu- ate radar propagation. Other factors, such as nonsimple reflecting interfaces, inadequate dielectric contrast, dominating dielectric contributors (e.g., water), and scattering, may also reduce the quality of the return signal detected at the radar receiver and ultimately affect the success of the radar's application.

During a field study in the Great Victoria Desert (GVD) of South Australia, GPR was applied as an aid to geochemical exploration to determine the stratigraphy of the shallow regolith (Lintern et al., 2009). A surprising outcome in the GPR data was its lack of penetration through the regolith. The GPR data required more than the usual gain adjustment and basic signal processing to preserve the amplitude variations of reflectivity that mark the target (calcrete) boundaries. As the signal was often at the level of residual systematic noise, a long interval 2D subtraction filter was used to remove the relatively strong bands of system noise and reveal the reflectivity variations. While the calcrete itself seemed fairly transparent, although irregular, it appeared that the overlying dry quartz sand ( $>90 \%$ silica) strongly attenuated the GPR signal. Our initial observations were supported by additional field tests at a large sand dune near to the GVD exploration area. These field tests confirmed that the sand was responsible for attenuating the signal. We therefore investigated the petrophysical properties of the GVD sands to find the unexpected cause of the attenuation.

After the GVD field trials, the GPR data collected were compared to the radar results from a dune near Perth, Western Australia [Perth quartz sand (PQS)] (Figure 1). It was evident that the useful depth of investigation of the radar data in the GVD area was significantly less, at $1 \mathrm{~m}$ compared to approximately 10-m depth in the PQS environment. Visual comparison of the GVD and PQS sand indicated that an obvious difference between the samples was an iron-oxideclay coating on the quartz which reddened (hue 5 YR yellowish red) the GVD sand samples when compared with the PQS samples. The quartz is aeolian derived from sand deposits to the west of the field site (Pell et al., 1999) and the iron oxide in the coating appeared to represent a very small proportion of the bulk sample. We

\footnotetext{
Manuscript received by the Editor 3 October 2010; revised manuscript received 16 February 2011; published online 18 November 2011

${ }^{1}$ CSIRO Earth Science and Resource Engineering, Bentley, Western Australia. E-mail: matthew.josh@csiro.au; mel.lintern@csiro.au; michael.verrall@ csiro.au.

${ }^{2}$ Curtin University, Exploration Geophysics, Kensington, Western Australia. E-mail: A.Kepic@curtin.edu.au.

(c) 2011 Society of Exploration Geophysicists. All rights reserved.
} 
hypothesized that iron group minerals (oxides and oxy-hydroxide minerals) coating the quartz grains was adversely affecting the dielectric response of the sample, thereby reducing the ability for electromagnetic propagation through the sand and regolith materials. Although we expected the radar to perform well (based on the PQS result), poor electromagnetic propagation was preventing both proper imaging of the calcrete layer and determination of the depth of the unconformity beneath it.

The possibility that iron oxide minerals may cause electromagnetic attenuation/reflection has not escaped the attention of other GPR investigators, particularly in Martian exploration for subterranean water (Heggy, et al., 2001; Paillou et al., 2001; Pettinelli et al., 2005; Williams and Greeley, 2004), and generally for GPR researchers (Cassidy, 2008; Olhoeft and Capron, 1993; Olhoeft and Capron 1994; Van Dam et al., 2002). Likewise, Robinson et al. (1994) noted that magnetic properties interfere with the ability to properly determine soil moisture content from dielectric data. The different data sets published are for samples retrieved from the field in most cases, and Pettinelli et al. (2005) published an experimental data set for a Martian analog made from a mixture of quartz sand and magnetite. Most notably, Paillou et al. (2001) investigated a natural Martian analog from an arid region in the Republic of Djibouti. The GVD sands have $<0.5 \%$ by weight iron oxide minerals, whereas, higher concentrations of iron oxide (1.5\%, Cassidy (2008); 8-20\%, Paillou et al. (2001); 5\%, Pettinelli et al. (2005); $5 \%$, Robinson et al. (1994)) are cited in these studies. There are no published examples where GPR has been so strongly attenuated by so little iron oxide. None of these studies would predict that the GVD sands will attenuate strongly based upon losses from the magnetic mode of electromagnetic radiation.

Thus, the purpose of this study was (1) to verify that the differences in the performance of GPR in the two environments could be attributed to the bulk dielectric/magnetic response of the samples and (2) to determine whether the coating on the quartz grains in the GVD samples was dominating their bulk dielectric/magnetic response. We investigated the coating to determine its mineralogy

a)

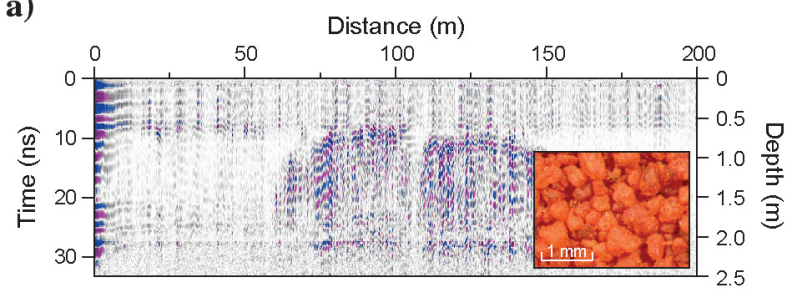

b)

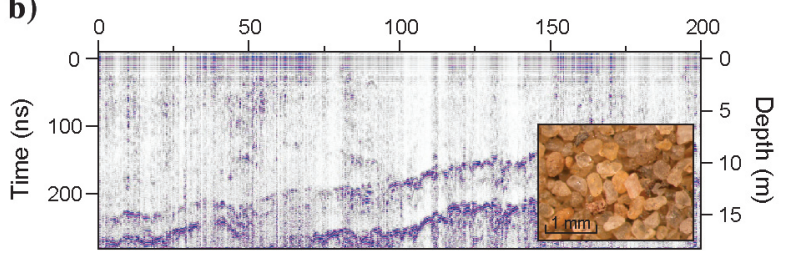

Figure 1. (a) An example radar image from the Great Victoria Desert (GVD) site with corresponding sample photo (inset). (b) Example radar image from a quartz sand dune near Perth, Western Australia with corresponding sample photo (inset). Note the difference in color of the samples and the order of magnitude difference in radar penetration. GVD data required significantly more processing to achieve the image provided which has led to additional artifacts. and the approximate weight percentage of the bulk sample that the coating represented.

\section{SAMPLE PREPARATION}

Four samples were collected from a soil pit at the GVD site (730 km northwest of Adelaide (South Australia) at $-30.89^{\circ} \mathrm{S}$ $\left.133.32^{\circ} \mathrm{E}\right)$ at different depths: TE90 $(0-20 \mathrm{~cm})$; TE91 $(20-40 \mathrm{~cm})$; TE92 $(40-60 \mathrm{~cm})$ and TE89 (the calcrete layer at 75-80 cm). We tested our hypothesis (that iron oxide was adversely affecting the GPR) by using a washing procedure to remove the iron oxide minerals from the coatings but leave the remaining coating minerals unaltered, and then we used dielectric and magnetic analysis to compare the samples before and after the washing process.

One PQS and four GVD samples were sieved to retain the $<250 \mu \mathrm{m}$ fraction so that the grains were sufficiently small enough to pack tightly into the dielectric measurement cell. Approximately $86 \%$ weight/weight $(\mathrm{w} / \mathrm{w})$ of the sample passed through this mesh size. This method was selected in preference to a grinding process to avoid destruction of the true in situ nature (i.e., uniform clay-coated quartz) of the sample. To remove the iron oxide content from the clay coating, but leave other components of the sample relatively unaffected, a partial extraction technique was employed. Approximately $5 \mathrm{~g}$ of the sample was warmed on a hot plate (to about $50^{\circ} \mathrm{C}$ ) with $50 \mathrm{~mL}$ of a mixture of $3 \mathrm{M} \mathrm{HCL}$ and $0.3 \mathrm{M}$ ascorbic acid for a period of one hour. The leach solution was prepared to reduce and dissolve mineral complexes from $\mathrm{Fe}^{3+}$ to $\mathrm{Fe}^{2+}$ (Mann and Lintern, 1984). Typically, easily reducible iron oxides, such as goethite, hematite, and maghemite, are dissolved. Resistate minerals, such as magnetite, ilmenite, and other strongly bound iron minerals, are unaffected, as are the clay minerals kaolinite, smectite, and illite/mica. The sand sample visibly changed color from a red to a gray color (similar to PQS) with a clear colorless liquor, indicating that the brown iron oxide minerals (goethite, hematite, and maghemite) had been reduced and dissolved leaving the sand and minor clay component unaffected. The sample was centrifuged and the liquor was decanted and discarded. The remaining sand sample was rinsed with deionized water, leaving a relatively iron oxide-free sample that was subsequently dried in ambient laboratory conditions before further analysis. Each sample was then reanalyzed in the dielectric cell along with the washed PQS.

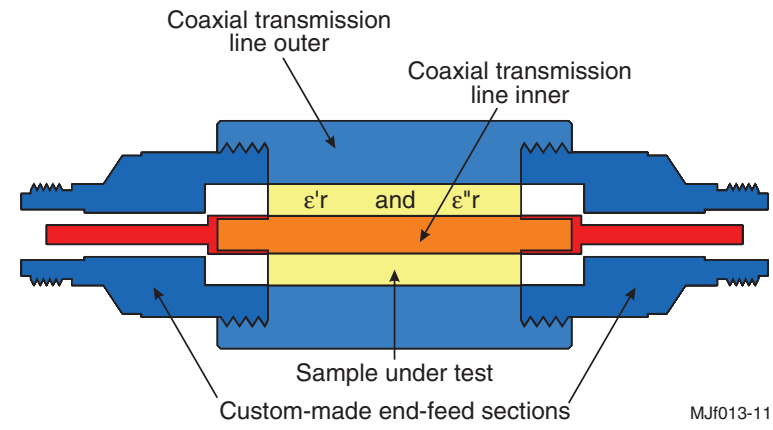

Figure 2. CSIRO 16-mm coaxial transmission line cell. The sample (yellow) is packed into the coaxial cavity formed between the inner (orange) and outer (light blue) conductors. The feed sections are attached to the cell and the measurement is done using an Agilent E5070B network analyzer. 


\section{METHODS}

\section{Dielectric analysis}

The dielectric analysis was performed on air-dried samples, so that the samples were as close as possible to their in situ state. The moisture content (determined by weighing and drying) of the GVD samples was $<1 \%$, which is consistent with the desert environment from which they were collected. Dielectric analysis was performed using a coaxial transmission line dielectric cell (Josh et al., 2009) developed according to the principles commonly used for dielectric spectroscopy (Baker-Jarvis et al., 1990; Nicholson and Ross, 1970; Von Hipel, 1954; Weir, 1974). The cell consisted of a 40-mm-long section of coaxial transmission line with inner and outer conductor diameters of $16 \mathrm{~mm}$ and $7 \mathrm{~mm}$ (Josh et al., 2009). The ends of the cell were coupled into conventional 50 -ohm coaxial cables by using

a)

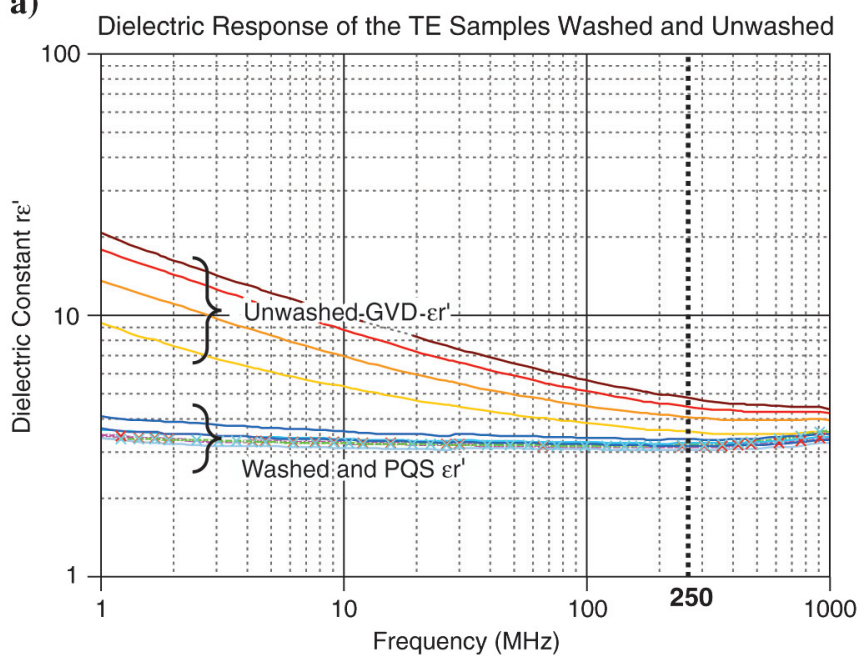

b)

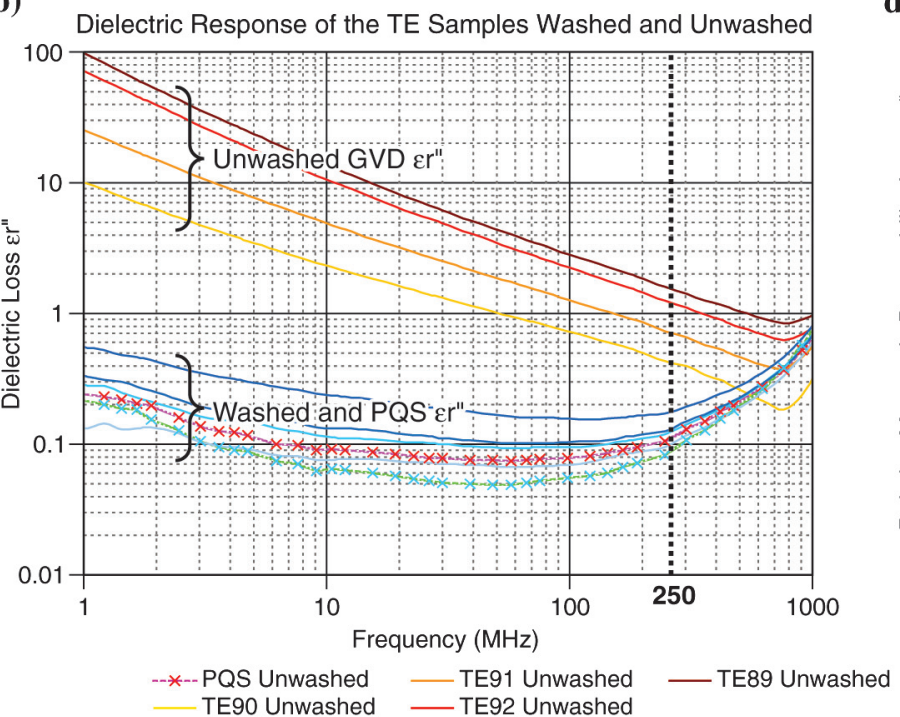

custom-made end-feed sections connected to an Agilent E5070B network analyzer to measure the reflection and transmission scattering parameters from $300 \mathrm{kHz}$ to $3 \mathrm{GHz}$ (Figure 2). Sample material was packed into the cell by using a packing funnel and then compacted with a mandrel. The reflection and transmission scattering parameters for the 16-mm coaxial transmission line dielectric cell that were provided by the network analyzer were inverted to determine the dielectric response of the sample. The inversion process was performed by using the Nicholson-Ross algorithm (Nicholson and Ross, 1970), to determine the dielectric response from $300 \mathrm{kHz}$ up to $3 \mathrm{GHz}$.

\section{Magnetic analysis}

The coaxial transmission line developed for dielectric analysis can also be used to calculate the magnetic permeability (Ohloeft c)

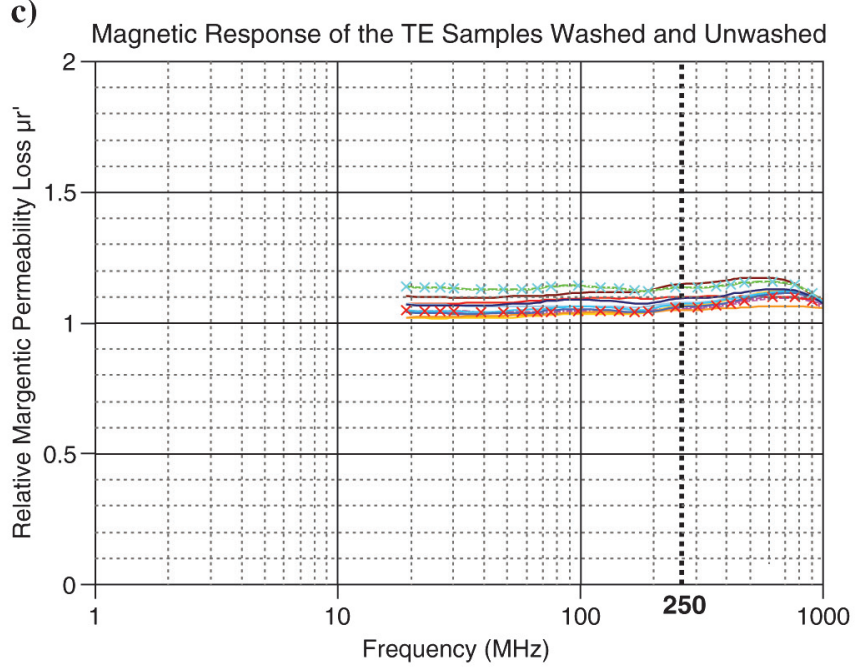

d)

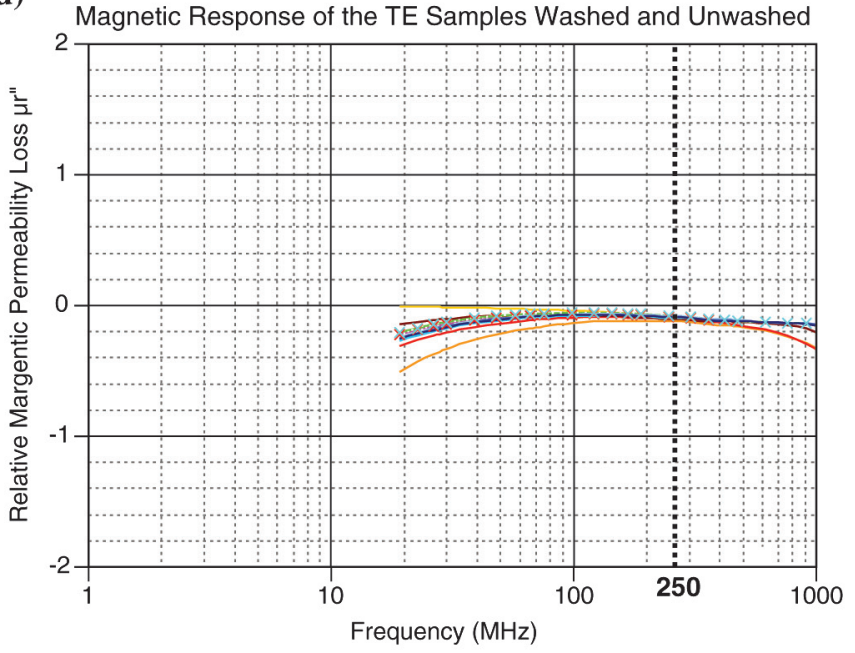

— TE91 Washed _ TE89 Washed

MJf010-11

Figure 3. ( $a$ and b) The real and imaginary components of the dielectric response of the quartz sand samples (GVD) with iron-rich coating before and after the acid-reductant washing to remove the iron. (c and d) The real and imaginary components of the relative magnetic permeability (determined by coaxial transmission line using Nicholson-Ross algorithm) of the quartz sand samples (GVD) with iron-rich coating before and after the acid-reductant washing to remove the iron. The dielectric and magnetic data have been color-coded into four groups. Red and orange lines plot the responses of the unwashed GVD samples, and blue and green lines plot the washed and PQS samples. The darker shades in each group represent the samples extracted from a greater depth. 
and Capron, 1993, 1994) and related losses via the Nicholson-Ross algorithm (Figure $3 \mathrm{c}$ and d), but our results indicate the samples are virtually nonmagnetic (real relative magnetic permeability $\mu_{r}{ }^{\prime}=$ 1.0, and imaginary relative magnetic permeability, $\mu_{r}{ }^{\prime \prime}=0.0$ with \pm 0.1 error); thus, magnetic losses appear to be insignificant. To obtain lower detection levels than the transmission line method, we used a hand held magnetic susceptibility instrument, a Bartington magnetic susceptibility meter (MS2B36, Bartington MS2 manual), that can provide approximately five decimal places of precision on low permeability materials, but is limited to two spot frequencies (465 Hz and $4650 \mathrm{~Hz}$ ). These measurements allow an upper bound for the magnetic permeability, and any associated losses, to be found as permeability decreases with frequency. The samples retrieved from the field sites were analyzed before and after washing in addition to two comparison laboratory-prepared mixtures of pure quartz sand $(99.9 \% \mathrm{w} / \mathrm{w})$ with magnetite and hematite that acted as a control $(0.1 \% \mathrm{w} / \mathrm{w})$, (Table 1$)$. None is very magnetic; thus, their ability to lose magnetic energy is less than the losses observed.

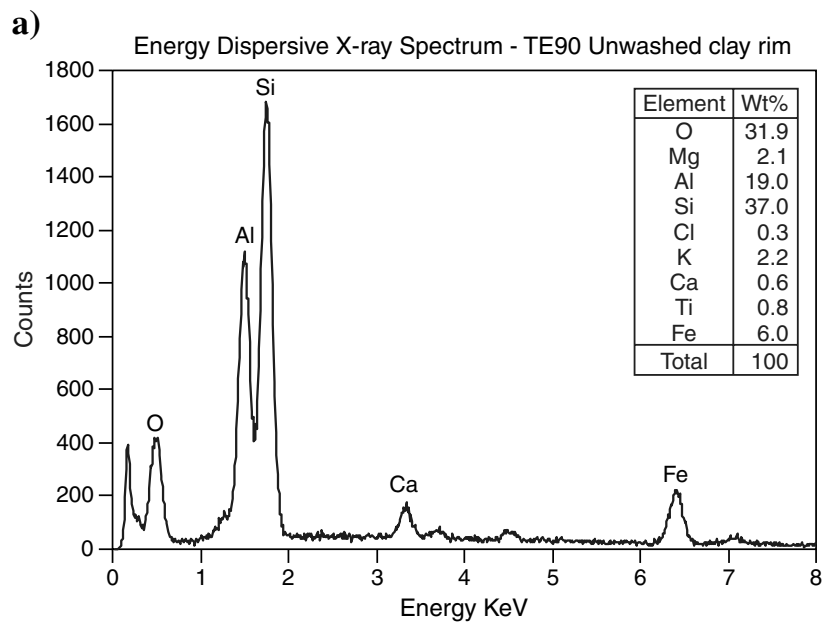

b)

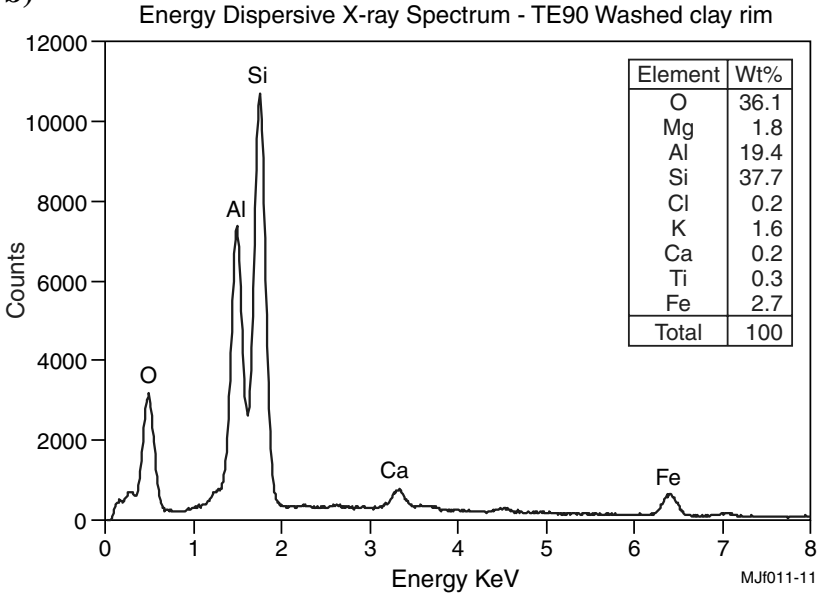

Figure 4. (a) SEM EDS spectrograms collected from the clay rim of the unwashed GVD sample, TE90. (b) SEM EDS spectrograms collected from the clay rim of the washed GVD sample, TE90. Note the reduction in total iron and calcium.

\section{Grain-coating mineralogy and geochemistry}

Polished sections were prepared by mounting a small quantity of the sample grain on a glass slide by using epoxy, and then grinding and polishing the sample to expose the cross section of the grains embedded in the epoxy with its coating rim intact. A Philips(FEI) XL40 controlled pressure scanning electron microscope (SEM) fitted with an EDAX energy dispersive X-ray spectrometer (EDS) was used to examine the polished sections of the sand grains and the composition of the coatings (Figure 4). Transmitted light optical microscopy was used on the polished sections to study the uniformity and thickness of the coatings and to make a visual estimate of percentage of coating in the sample. X-ray fluorescence (XRF, Ultratrace Laboratories Ltd, Western Australia) was used to quantify the total iron (and other major elements) in the samples. These analyses were done on all of the GVD samples (TE89-TE92) and the PQS before and after washing to monitor any changes that have taken place during the washing process. Results of the XRF analysis are presented in Table 2. A Philips X'pert X-ray diffractometer (XRD) fitted with cobalt X-ray tube was used to determine mineralogy. A portion of the sample was placed in a test tube with water in an ultrasonic bath for five minutes to separate the clay coatings from the quartz grains. This was then allowed to settle, and a $<2 \mu \mathrm{m}$ fraction was drawn off for testing.

\section{RESULTS AND DISCUSSION}

\section{Dielectric and magnetic behavior}

The untreated samples show an increase in real and imaginary dielectric permittivity with increasing depth. After the coating was altered using the acid wash, the real dielectric permittivity reduced across the frequency range. At the operating frequency of the radar used in this study $(250 \mathrm{MHz})$, the acid wash resulted in a reduction of the real dielectric permittivity to approximately $75 \%$ of its unwashed value, and a reduction of the imaginary dielectric permittivity to approximately $10 \%$ of its unwashed value. It is

Table 1. Magnetic permeability of the washed and unwashed samples at $465 \mathrm{~Hz}$ and $4650 \mathrm{~Hz}$.

\begin{tabular}{lcc}
\hline & $\mu^{\prime} r$ at $465 \mathrm{~Hz}$ & $\mu^{\prime} r$ at $4650 \mathrm{~Hz}$ \\
\hline PQS unwashed & 1.0002312 & 1.0002312 \\
PQS washed & 1.0002714 & 1.0002714 \\
TE89 unwashed & 1.0004222 & 1.0003921 \\
TE90 unwashed & 1.0005102 & 1.0004800 \\
TE91 unwashed & 1.0004851 & 1.0004851 \\
TE92 unwashed & 1.0004926 & 1.0004926 \\
TE89 washed & 1.0000578 & 1.0000578 \\
TE90 washed & 1.0000402 & 1.0000729 \\
TE91 washed & & \\
TE92 washed & 1.0000653 & 1.0000829 \\
Average unwashed GVD & 1.0004775 & 1.0004624 \\
Average washed GVD & 1.0000545 & 1.0000712 \\
0.1\% magnetite 99.9\% quartz & 1.0008520 & 1.0009098 \\
0.1\% hematite 99.9\% quartz & 1.0000251 & 1.0000176 \\
\hline
\end{tabular}


also evident that both the real and imaginary dielectric response of the unwashed GVD samples are much higher than all other samples tested, including the washed GVD samples.

The magnetic permeability is essentially unaffected by the minor presence of reducible iron oxide minerals in the samples. The two control samples containing $0.1 \%$ magnetite and $0.1 \%$ hematite are consistent with published expected values whereby magnetite is strongly magnetic and hematite is not. The control sample containing magnetite has the highest magnetic permeability of all the samples tested despite having only $0.1 \%$ magnetite. Our data shows that the GVD samples are essentially nonmagnetic $\left(\mu_{r}{ }^{\prime}=1\right.$ and $\mu_{r}{ }^{\prime \prime}=0$ ), based on the limited precision available with transmission line measurement and circumstantial evidence that $\mu_{r}{ }^{\prime \prime}$ cannot be nonzero when $\mu_{r}{ }^{\prime}$ is so precisely shown to be almost exactly 1 . Therefore, the magnetic permeability did not affect the GPR performance.

\section{Determination of attenuation factor}

The electromagnetic wave attenuation factor $(\beta)$ was calculated using knowledge of the magnetic permeability $\mu$, the electrical permittivity $\varepsilon$, and the angular frequency $\omega$ by using (Stratton, 1941):

$$
k^{2}=\mu \varepsilon \omega^{2}+j \mu \sigma \omega
$$

where the complex magnetic relative permeability and dielectric relative permittivity are used:

$$
\begin{gathered}
\varepsilon=\varepsilon_{o}\left(\varepsilon_{r}^{\prime}-j \varepsilon_{r}^{\prime \prime}\right) \\
\mu=\mu_{o}\left(\mu_{r}^{\prime}-j \mu_{r}^{\prime \prime}\right)
\end{gathered}
$$

Using

$$
k=\alpha+j \beta \Rightarrow k^{2}=\alpha^{2}-\beta^{2}+2 j \alpha \beta
$$

(where $\beta$ is the attenuation factor). Equation 1 becomes:

$$
\begin{aligned}
k^{2}= & \mu_{o} \varepsilon_{o} \omega^{2}\left(\mu_{r}^{\prime} \varepsilon_{r}^{\prime}-\mu_{r}^{\prime \prime} \varepsilon_{r}^{\prime \prime}\right)+\mu_{o} \mu_{r}^{\prime \prime} \omega \sigma \\
& +j\left(\mu_{o} \mu_{r}^{\prime}-\mu_{o} \varepsilon_{o} \omega^{2}\left(\mu_{r}^{\prime \prime} \varepsilon_{r}^{\prime}+\mu_{r}^{\prime} \varepsilon_{r}^{\prime \prime}\right)\right)
\end{aligned}
$$

Equating real and imaginary coefficients from equations 4 and 5 gives:

$$
\begin{aligned}
& \beta= \\
& \quad \sqrt{\frac{\mu_{o} \omega}{2}\left\{\sqrt{\left(\mu_{r}^{\prime 2}+\mu_{r}^{\prime \prime 2}\right)\left(\varepsilon_{o}^{2} \omega^{2}\left(\varepsilon_{r}^{\prime 2}+\varepsilon_{r}^{\prime \prime 2}\right)+\sigma^{2}-2 \varepsilon_{o} \varepsilon_{r}^{\prime \prime} \omega \sigma\right)}-\left(\varepsilon_{o} \omega\left(\mu_{r}^{\prime} \varepsilon_{r}^{\prime}-\mu_{r}^{\prime \prime} \varepsilon_{r}^{\prime \prime}\right)+\sigma \mu_{r}^{\prime \prime}\right)\right\}}
\end{aligned}
$$

\begin{tabular}{|c|c|c|c|c|c|c|c|c|c|c|}
\hline & TE90_U & TE90_W & TE91_U & TE91_W & TE92_U & TE92_W & TE89_U & TE89_W & PQS_U & PQS_W \\
\hline $\mathrm{Fe} \%$ & 0.406 & 0.231 & 0.434 & 0.252 & 0.424 & 0.245 & 0.389 & 0.259 & 0.501 & 0.455 \\
\hline $\mathrm{Al} \%$ & 1.625 & 2.042 & 1.741 & 1.921 & 1.672 & 1.963 & 1.519 & 2.085 & 1.064 & 0.862 \\
\hline $\mathrm{Ca} \%$ & 0.071 & 0.007 & 0.786 & 0.036 & 0.900 & 0.043 & 4.257 & 0.064 & 0.150 & 0.007 \\
\hline $\mathrm{Si} \%$ & 42.900 & 42.467 & 41.754 & 42.458 & 41.524 & 42.430 & 36.634 & 41.929 & 42.182 & 42.798 \\
\hline
\end{tabular}

Our laboratory instruments measure conductivity as part of the ima-

\begin{tabular}{|c|c|c|c|c|c|}
\hline & $\varepsilon^{\prime} r$ & $\varepsilon^{\prime \prime} r$ & $\mu^{\prime} r(4650 \mathrm{~Hz})$ & $\beta\left(\mathrm{m}^{-1}\right)$ & $\beta\left(\mathrm{dBm}^{-1}\right)$ \\
\hline PQS unwashed & 3.15 & 0.106 & 1.0002312 & 0.156 & 1.358 \\
\hline PQS washed & 3.18 & 0.0827 & 1.0002714 & 0.121 & 1.054 \\
\hline TE89 unwashed & 4.86 & 1.61 & 1.0003921 & 1.888 & 16.389 \\
\hline TE90 unwashed & 3.60 & 0.435 & 1.0004800 & 0.600 & 5.204 \\
\hline TE92 unwashed & 4.52 & 1.258 & 1.0004926 & 1.536 & 13.330 \\
\hline TE89 washed & 3.35 & 0.170 & 1.0000578 & 0.243 & 2.111 \\
\hline TE90 washed & 3.06 & 0.0950 & 1.0000729 & 0.142 & 1.235 \\
\hline TE91 washed & 3.24 & 0.118 & 1.0000000 & 0.172 & 1.490 \\
\hline TE92 washed & 3.17 & 0.125 & 1.0000829 & 0.184 & 1.596 \\
\hline
\end{tabular}
ginary relative permittivity $\varepsilon_{r}{ }^{\prime \prime}$, therefore, the conduction term $\sigma$ can be set to zero. The attenuation factor becomes:

$$
\beta=\omega \sqrt{\frac{\mu_{o} \varepsilon_{o}}{2}\left\{\sqrt{\left(\mu_{r}^{\prime 2}+\mu_{r}^{\prime \prime 2}\right)\left(\varepsilon_{r}^{\prime 2}+\varepsilon_{r}^{\prime 2}\right)}-\left(\mu_{r}^{\prime} \varepsilon_{r}^{\prime}-\mu_{r}^{\prime \prime} \varepsilon_{r}^{\prime \prime}\right)\right\}}
$$

Table 2. Content of the principle elements of interest determined by XRF for the washed (W) and unwashed (U) samples.

Table 3. Attenuation calculated from the dielectric and magnetic response of washed and unwashed samples at $250 \mathrm{MHz}$. 


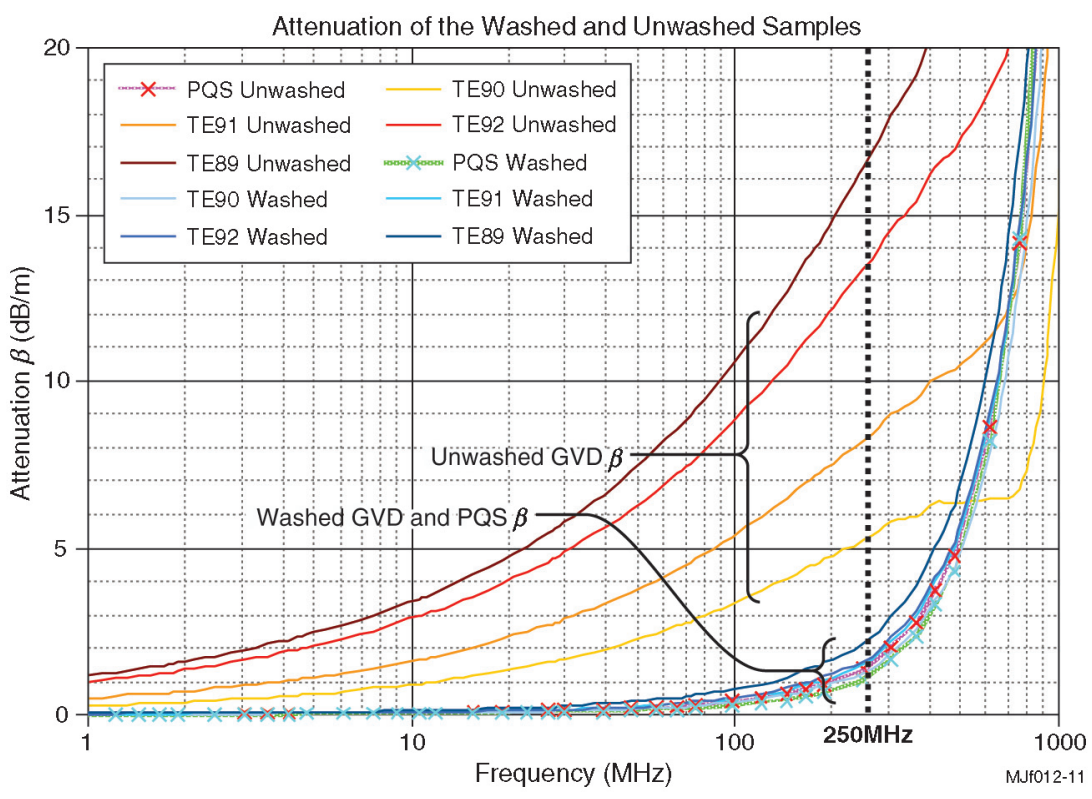

Figure 5. The attenuation factor of the quartz sand samples (GVD and PQS) with ironrich coating before and after the acid-reductant washing to remove the iron. a)

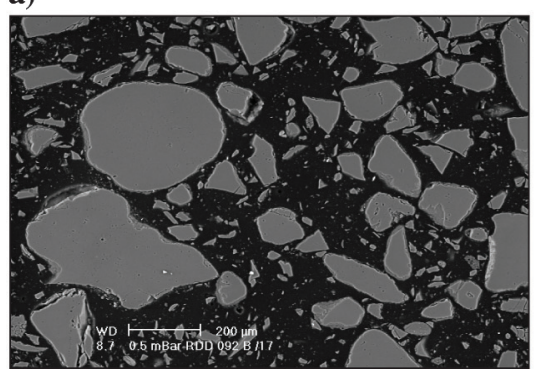

b)

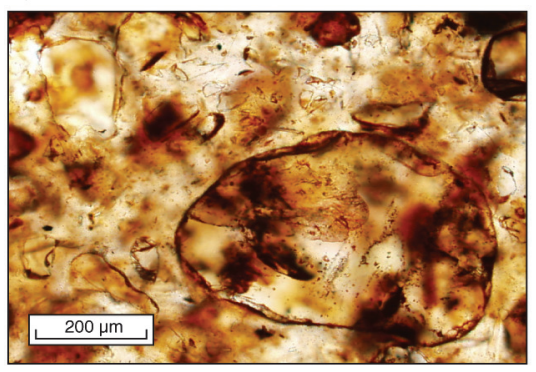

c)

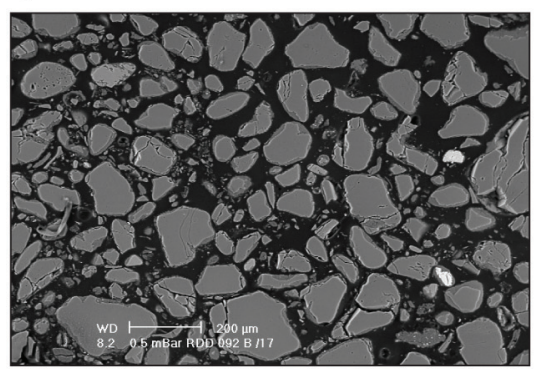

d)

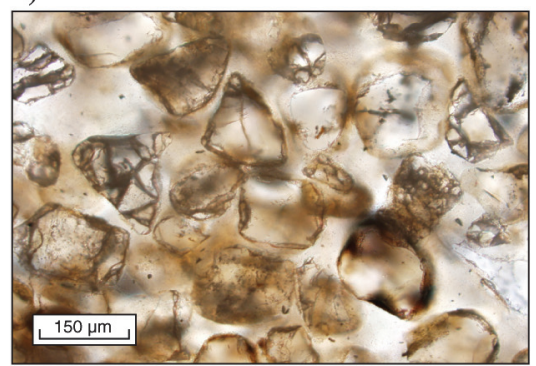

e)

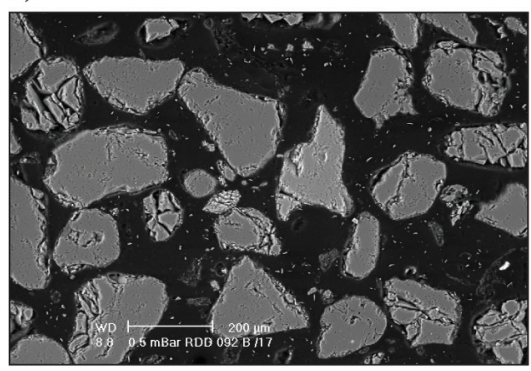

f)

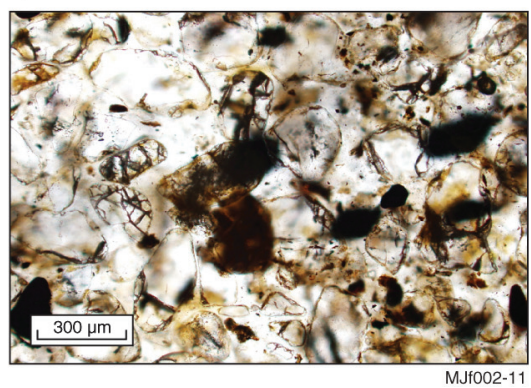

Figure 6. SEM (left) and transmitted light (right) images of (a and b) unwashed GVD sand (TE90); (c and d) washed GVD sand (TE90); and (e and f) unwashed PQS. where the real and imaginary relative permittivity $\varepsilon_{r}{ }^{\prime \prime}$ and $\varepsilon_{r}{ }^{\prime \prime}$ were provided from dielectric data at $250 \mathrm{MHz}$ (Figure 3). Real relative permeability $\mu_{r}{ }^{\prime}$ was provided by the Bartington magnetic susceptibility meter (Table 1) and imaginary relative permeability $\mu_{r}{ }^{\prime \prime}$ was assumed to be zero (explained above). $\varepsilon_{\mathrm{o}}$ is the permittivity of free space $(8.85 \mathrm{pF} / \mathrm{m})$ and $\mu_{\mathrm{o}}$ is the permeability of free space $\left(4 \pi \times 10^{-7} \mathrm{H} / \mathrm{m}\right)$. Values $\beta$ were calculated using equation 7 and presented in Table 3 and Figure 5.

\section{Grain-coating mineralogy and geochemistry}

Washing has reduced the iron content of the coating on the sand from $6.0 \%$ to $2.7 \% \mathrm{w} / \mathrm{w}$, and the calcium content from $0.6 \%$ to $0.2 \% \mathrm{w} / \mathrm{w}$. Aluminum, a clay indicator (Pardue et al., 1992) remains substantially unchanged in the coating (19.0\% to $19.4 \%$ ), indicating that the clay minerals were largely unaffected by the washing process. The optical images in Figure 6 indicate that the coating is still present, but the obvious change in color from dark red to a whitish color supports the SEM mineralogy analysis observation of a reduction in iron oxides.

The surface of quartz grains in both the washed and unwashed GVD (TE90) samples have a coating on most of the grains with approximately uniform thickness. It is therefore apparent that the washing process only removed the iron within the clay coating but left the coating otherwise unaffected. It is also visually evident from the grain cross sections that the coating material represents a small fraction of the bulk volume of the sample. The optical image of the transmitted light through the sample confirms that the red color of the sample is entirely associated with the coating on the quartz grains and that the washing process has removed the component responsible for the red coloration. The PQS sample does not contain any visually significant coating on the quartz grains.

XRD examination on the clay fraction indicates that it is composed of mostly kaolinite with minor amounts of smectite. The washing process did not shift the peaks in the XRD spectra or the apparent crystal structure of the clays. However, it did reduce the concentrations of iron and calcium (determined by using XRF). Iron concentration in the GVD samples was reduced from approximately $0.4 \% \mathrm{w} / \mathrm{w}$ to approximately $0.25 \% \mathrm{w} / \mathrm{w}$. It is remarkable that this small concentration has such a profound color influence on the GVD samples. Although the concentration of the iron oxide minerals was below the detection limit of the XRD and the individual minerals could not be quantified, the color change from strongly red to gray that occurred during washing 
confirmed that iron oxide minerals, e.g., hematite, goethite, and maghemite had been removed. The PQS samples also contained iron according to the XRF analysis, but this sample was not red in color, and almost certainly contained much lower concentrations of nonresistate iron minerals than the GVD samples. The remaining iron in the samples after washing exists in resistate minerals, e.g., magnetite and ilmenite. The calcium reduction was caused by dissolving of calcite by the acid (especially for the highly calcareous sample TE89). Aluminum (clay) and silicon (quartz) have both increased to mass balance for the loss of the other material therefore all other changes in the observed electromagnetic response of the samples are related to either the calcium or iron.

Sample TE92 had less than $1 \%$ calcite prior to washing; and after washing, its real dielectric permittivity (Figure 3a) has reduced by approximately a factor of two, and its imaginary dielectric permittivity has reduced by a factor of approximately 10. A comparable change was also observed for sample TE89 that had a relatively high calcium content of over $4 \%$. Thus, the calcium content had little if any influence. In contrast, the iron oxide in the coating of the grains has increased the radar signal attenuation by approximately a factor of seven and led to the reduction in the original radar performance. From the XRF data, this iron oxide constitutes approximately $0.15 \%$ of the bulk of the sample. It is apparent that only a small percentage of iron oxide minerals contained within the clay coating contributes to the observed reduction in radar performance and that other iron (approximately $0.25 \%$ of the total sample content) bound in the resistate or silicate phases is not a major contributor to the reduction in radar performance.

\section{CONCLUSIONS}

It is common for GPR practitioners to attribute poor performance of their instruments to the presence of clay minerals attenuating the radar signals. In this study, we have found that the presence of reducible iron oxide minerals because grain coatings also provide a surprisingly significant reduction in GPR performance. We have investigated sand samples from a geophysical survey site in the Great Victoria Desert (Australia) where GPR imaging performed below expectation. Samples from the field site contained more than $90 \%$ pure quartz and less than $1 \%$ moisture, which would usually be considered an ideal environment for radar imaging; however, preliminary dielectric analysis of the samples verified that the GPR attenuation factor was 10 times that of regular quartz sand. Acid washing the samples to remove the reducible iron oxide minerals was successful in significantly altering the dielectric behavior of the GVD samples so as to become more similar in behavior and appearance to other quartz sand samples (PQS). XRD analysis indicates that the washing process did not alter the mineralogy or content of the minor clay component of the samples; thus, clay minerals are not responsible for the anomalous attenuation. XRF analyses showed that both calcium and iron were lost during the washing process; however, sample TE89 with very high calcium showed a similar change in dielectric behavior compared with the other samples with negligible calcium. This suggests that the minor component of iron oxide, exclusive of all other constituents, has led to the change in dielectric behavior of the GVD sand sample and, therefore, to the poor performance of the GPR. We therefore conclude that the depth of penetration of the GPR was reduced by a factor of 7 as a result of geological constituents representing less than $0.15 \%$ of the total element content.

\section{ACKNOWLEDGMENTS}

The authors would like to thank Ben Clennell for his advice on petrophysics procedures; Tony Siggins for his advice in radar image interpretation and radar performance relating to dielectrics; Elmar Strobach for his assistance in providing sample data sets, photographs, and recommended texts; Greg Hitchen for SEM image acquisition; Peter Eadington and David Mills for optical image acquisition. We would also like to thank David Annetts, Lionel Esteban, Gary Olhoeft, two additional anonymous reviewers, and the associate editor for their helpful comments.

\section{REFERENCES}

Baker-Jarvis, J., E. J. Vanzura, and W. A. Kissick, 1990, Improved technique for determining complex permittivity with the transmission reflection method: IEEE Transactions on Microwave Theory and Techniques, 38, no. 8, 1096-1103, doi: 10.1109/22.57336.

Cassidy, N. J., 2008, Frequency-dependent attenuation and velocity characteristics of nano-tomicroscale, lossy, magnetite-rich materials: Near Surface Geophysics, 6, 6, 341-355.

Daniels, D. J., D. J. Gunton, and H. F. Scott, 1988, Introduction to subsurface radar: IEEE Proceedings F, Radar and Signal Processing, 135, 4, 278-320, doi: 10.1049/ip-f-1.1988.0038.

Davis, J. L., and A. P. Annan, 1989, Ground-penetration radar for highresolution mapping of soil and rock stratigraphy: Geophysical Prospecting, 37, 531-551, doi: 10.1111/gpr.1989.37.issue-5.

Doolittle, J. A., and M. E. Collins, 1995, Use of soil information to determine application of ground penetrating radar: Journal of Applied Geophysics, 33, 101-108.

Josh, M., B. Clennell, and T. Siggins, 2009, Practical broadband dielectric measurement of geological samples: 50th SPWLA Annual Logging Symposium.

Lintern, M. J., A. W. Kepic, and M. Josh, 2009, Using ground penetrating radar to delineate sub-surface calcrete in the Great Victoria Desert, South Australia: Implication for gold exploration: 24th International Applied Geochemistry Symposium, 1022-1024.

Mann, A. W., and M. J. Lintern, 1984, Field analysis of heavy metals by portable digital voltammeter: Journal of Geochemical Exploration, 22, 333-348, doi: 10.1016/0375-6742(84)90018-9.

Nicholson, A. M., and G. F. Ross, 1970, Measurement of the intrinsic properties of materials by time-domain techniques: IEEE Transactions on Instruments and Measurement, IM-19, no. 4, 377-382, doi: 10.1109/TIM .1970 .4313932 .

Olhoeft, G. R., and D. E. Capron, 1993, Laboratory measurements of the radiofrequency electrical and magnetic properties of soils from near Yuma, Arizona: U.S. Department of Interior USGS Open File Report, 93-701.

Olhoeft, G. R., and D. E. Capron, 1994, Petrophysical causes of electromagnetic dispersion: Proceedings of the Fifth International Conference on Ground Penetrating Radar, Kitchener, 1, 145-152.

Paillou, P., G. Grandjean, J. M. Malezieux, G. Ruffie, E. Heggy, D. Piponnier, P. Dubois, and J. Achache, 2001, Performances of ground penetrating radars in arid volcanic regions: Consequences for Mars subsurface exploration: Geophysical Research Letters, 28, no. 5, 911-914, doi: 10.1029/1999GL008449.

Pardue, J. H., R. D. Delaune, and W. H. Patrick Jr., 1992, Metal to aluminum correlation in Louisiana coastal wetlands: identification of elevated metal concentrations: Journal of Environmental Quality, 21, 539545, doi: 10.2134/jeq1992.00472425002100040003x.

Pell, S. D., A. R. Chivas, and I. S. Williams, 1999, Great Victoria Desert: Development and sand provenance: Australian Journal of Earth Sciences, 46, 289-299.

Pettinelli, E., G. Vannaroni, A. Cereti, A. R. Pisani, F. Paolucci, D. Del Vento, D. Dolfi, S. Riccioli, and F. Bella, 2005, Laboratory investigations into the electromagnetic properties of magnetite/silica mixtures as Martian soil stimulants: Journal of Geophysical Research, 110, no. E04013.

Robinson, D. A., J. P. Bell, and C. H. Batchelor, 1994, Influence of iron minerals on the determination of soil water content using dielectric 
techniques: Journal of Hydrology, 161, 169-180, doi: 10.1016/0022-1694 (94)90127-9.

Stratton, J. A., 1941, Electromagnetic theory: McGraw-Hill Book Co., 276.

Van Dam, R. L., W. Schlager, M. J. Dekkers, and J. A. Huisman, 2002, Iron oxides as a cause of GPR reflections: Geophysics, 67, 536-545, doi: 10 $.1190 / 1.1468614$

Von Hipel, A. R., 1954, Dielectric materials and their applications: The Technology Press of MIT.
Weaver, W., 2006, Ground penetrating radar mapping in clay: Success from South Carolina, USA: Archeological Prospection, 13, 147-150, doi: 10 .1002/(ISSN)1099-0763.

Weir, W. B., 1974, Automatic measurement of complex dielectric constant and permeability at microwave frequencies: Proceedings of the IEEE, 62, 33-36, doi: 10.1109/PROC.1974.9382.

Williams, K. K., and R. Greeley, 2004, Measurements of dielectric loss factors due to a Martian dust analog: Journal of Geophysical Research Planets: JGR, 109, no. E10006. 